\title{
Chromosome numbers in the genus Mimosa L.: cytotaxonomic and evolutionary implications
}

\author{
Nair Dahmer - Marcelo F. Simon • Maria Teresa Schifino-Wittmann • \\ Colin E. Hughes $\cdot$ Silvia Teresinha Sfoggia Miotto $\cdot$ \\ Julio Cesar Giuliani
}

Received: 9 March 2010/Accepted: 4 October 2010/Published online: 19 November 2010

(C) Springer-Verlag 2010

\begin{abstract}
Chromosome numbers were determined for 125 accessions of 92 taxa of Mimosa from all five of Barneby's (Mem New York Bot Gard 65:1-835, 1991) taxonomic sections. For 69 species, 1 subspecies and 8 varieties, chromosome numbers are presented for the first time, for 6 species and 1 variety previously published data have been confirmed and for 3 species and 2 varieties different numbers were found. Results show that $74 \%$ of the accessions were diploid $(2 n=2 x=26)$ and $26 \%$ polyploid, these mostly tetraploid $(2 n=4 x=52)$ but with two triploid $(2 n=3 x=39)$. These results double the number of Mimosa species for which the chromosome count is known from less than $10 \%$ previously reported to more than $20 \%$, representing an important advance in the
\end{abstract}

N. Dahmer · M. T. Schifino-Wittmann $(\bowtie) \cdot$ J. C. Giuliani Departamento de Plantas Forrageiras e Agrometeorologia, Faculdade de Agronomia, UFRGS, Caixa Postal 15100, Porto Alegre, RS 91501-970, Brazil

e-mail: mtschif@ufrgs.br

M. F. Simon

EMBRAPA Recursos Genéticos e Biotecnologia, Caixa Postal 02372, Brasília, DF 70770-917, Brazil

C. E. Hughes

Department of Plant Sciences, University of Oxford, South Parks Road, Oxford OX1 3RB, UK

Present Address:

C. E. Hughes

Institut für Systematische Botanik, Zollikerstrasse 107, 8008 Zurich, Switzerland

\section{S. T. S. Miotto}

Departamento de Botânica, Instituto de Biociências, Av. Bento Gonçalves 9500, prédio 43433,

Porto Alegre, RS 91501-970, Brazil cytotaxonomy of this legume genus. These results together with literature data show that ca. $78 \%$ of Mimosa species are diploid. Polyploids are present in most of the taxonomic sections and in different lineages across the genus. No particular chromosome number is restricted to a given section or lineage. A possible relation between geography, species distribution, polyploidy and invasiveness was detected, however, further studies based on more accessions, especially from higher latitudes, are required before firm conclusions can be drawn.

Keywords Mimosa - Chromosome numbers . Cytotaxonomy · Evolution - Intraspecific variability . Leguminosae

\section{Introduction}

The genus Mimosa L. (Fabaceae-Mimosoideae) comprises around 530 species (ca. 704 taxa), ca. 461 native to the Americas, 31 to Madagascar (mostly endemics), 4 native to east Africa and 4 to southwest Asia (Barneby 1991; Du Puy et al. 2002; Simon 2009). Most of the species occur in the tropics, at low and medium altitudes, but some reach subtropical or warm temperate regions of the U.S.A. in the north and Argentina and Uruguay in the south. Their habitats range from equatorial macrothermic forests to fireprone tropical savannas, subtropical grasslands, seasonally dry tropical and subtropical forests and thorny scrub vegetation, deserts and many ruderal sites including tropical pastures, with notable adaptations in growth habit across these diverse environments (Barneby 1991; Lewis et al. 2005). Brazil harbours the greatest concentration of species in the genus, especially in the Cerrado region, a zone of high biodiversity, where a quarter of all Mimosa species are 
found, with $74 \%$ of these restricted to the Cerrado biome and around 50\% endemic (Simon and Proença 2000).

The origin of the genus was probably in the humid regions of South America (Polhill et al. 1981). The main species diversity centres are central Brazil and central and southern Mexico. Smaller centres are located in the Caribbean, espcially Cuba-Hispaniola (Dominican Republic and Haiti), the Orinoco basin and Madagascar (Barneby 1991; Du Puy et al. 2002).

Many species of Mimosa are economically important, such as M. scabrella Benth., a pioneer tree species in the araucária forests of southern Brazil that may reach $15-20 \mathrm{~m}$ in height. It is cultivated for shade in coffee plantations, and the wood is used in building and carpentry, and for cellulose and fuel (Barneby 1991; Caramori et al.1996). Other species are used as living fences (M. caesalpiniifolia Benth), in the cosmetic industry and in traditional folk medicine [M. tenuiflora (Wild.) Poir.] (Camargo-Ricalde et al. 2001; Lorenzi 2000a; Rivera-Arce et al. 2007). Many Mimosa species, due to their nitrogen fixation ability, are also used for soil enrichment and recuperation of degraded land (Dhillion and CamargoRicalde 2005). Some other species such as M. pigra L. (Lowe et al. 2000) and M. pudica L. (Lorenzi 2000b) are among the world's most aggressive invaders outside their native range and are nowadays widespread around the world.

The first critical classification of Mimosaceae and Mimosa was by Bentham in 1841 and 1875 and, despite new classification proposals such as that by Britton and Rose in 1928, Bentham's main definitions were maintained by Barneby (1991) in his comprehensive and widely used infrageneric classification and taxonomic revision of Mimosa. Barneby (1991) recognised five sections [Mimadenia (ca. 15 species), Batocaulon (190 species), Habbasia (ca. 78 species), Calothamnos (ca. 26 species) and Mimosa (with ca. 177 species and several subspecies and varieties)], subdivided in 41 series. According to Barneby (1991) and two recent phylogenetic studies (Bessega et al. 2008; Simon et al. 2009) Mimadenia is sister to the other species of the genus, and section Mimosa is the most derived group and also the section with the most complicated taxonomy. In the densely sampled phylogenetic analysis (Simon 2009; Simon et al. 2009) including 259 species representing all five sections and 37 out of the 41 series proposed by Barneby (1991), it is also clear that while the rest of Barneby's (1991) sections do not form natural monophyletic groups, there is broad overall congruence with Barneby's vision of the genus with many of the other infrageneric groups (series and subseries) recovered as monophyletic.

Although progress is being made towards a new phylogenetic classification of Mimosa, as a new infrageneric classification for Mimosa has not yet been formally proposed, in this paper we will follow Barneby's (1991) monograph.

Chromosome numbers have been reported for around 67 Mimosa taxa, representing less than $10 \%$ of all taxa (Federov 1969; Alves and Custódio 1989; Seijo 1993, 1999, 2000; Seijo and Fernández 2001; IPCN 2009). Most of them are diploid with $2 n=2 x=26$ chromosomes. A few are tetraploid $(2 n=4 x=52)$ and even fewer are octaploid $(2 n=8 x=104)$. Goldblatt (1981) suggested $x=14$ as the basic number in Mimosoideae, and therefore $x=13$, found in Mimosa and other genera, would probably have been derived by disploidy. In a few cases, there are reports of intraspecific variability in ploidy levels (mostly diploid and tetraploid individuals) as in $M$. pudica (IPCN), M. somnians Humb. \& Bonpl. ex Willd, M. debilis H.B. ex Willd.(Seijo 1993, 1999, 2000) and M. balansae Micheli (Seijo and Fernández 2001). For the majority of the species with chromosome counts, only one or a few populations have been analysed. Therefore, the absence of intraspecific variation in ploidy levels could be due to undersampling within the geographic range of a given species.

This work aimed at analysing chromosome numbers in a range of Mimosa species in order to broaden the cytogenetic information of this large genus and to investigate the importance of chromosome numbers as a cytotaxonomic character and the role of polyploidy in Mimosa evolution.

\section{Materials and methods}

Seeds of 125 accessions of 92 Mimosa taxa were collected mainly from the Brazilian Cerrado region but also from some other regions of Brazil, Uruguay, Equador, Bolivia, Peru, Honduras, Panama, Mexico, the United States of America, Taiwan, India, Nepal, Burkina Faso and Madagascar (Table 1). Most of the accessions were collected by the authors while a few were obtained from the Millenium Seed Bank (UK), seed companies and other collaborators. Taxonomic vouchers of the accessions are deposited at the following herbaria: FHO (Oxford University, UK), K (Kew, Royal Botanic Gardens, UK), CEN (Embrapa Recursos Genéticos e Biotecnologia, Brazil), UB (Universidade de Brasília, Brazil), RB (Jardim Botânico do Rio de Janeiro, Brazil), UAMIZ (Universidad Autónoma Metropolitana, Mexico), MEXU (Herbario Nacional, Mexico), HUEFS (Universidade Estadual de Feira de Santana, Brazil) and ICN (Departamento de Botânica Universidade Federal do Rio Grande do Sul, Brazil).

Somatic chromosome numbers were determined in root tip cells. Seeds were scarified by a small cut in the testa and germinated in petri dishes with moist filter paper. When the roots were around $1 \mathrm{~cm}$ in length, they were pretreated with a saturated solution of paradichlorobenzene for $24 \mathrm{~h}$ 
Table 1 Accessions and taxa of Mimosa analysed, taxonomic section according to Barneby (1991), place and coordinates of collection and chromosome number

\begin{tabular}{|c|c|c|c|c|c|}
\hline Species & Section & Accession & Place of collection & Coordinates & Chromosome no. \\
\hline $\begin{array}{l}\text { M. acantholoba (Willd.) } \\
\text { Poir. var. } \\
\text { acantholoba }\end{array}$ & Batocaulon & Sarkinen, T.E. 2150 & Peru, Piura, Morropon & $5^{\circ} 13^{\prime} \mathrm{S}, 80^{\circ} 00^{\prime} \mathrm{W}$ & 26 \\
\hline $\begin{array}{l}\text { M. acutistipula Benth. } \\
\text { var. acutistipula }{ }^{\mathrm{a}}\end{array}$ & Mimosa & Way, M.J. 2438 & $\begin{array}{l}\text { Brazil, Pernambuco, } \\
\text { Petrolina }\end{array}$ & $8^{\circ} 52^{\prime} \mathrm{S}, 40^{\circ} 45^{\prime} \mathrm{W}$ & 52 \\
\hline M. adenocarpa Benth. ${ }^{\mathrm{a}}$ & Batocaulon & Coradin, L. 8532 & Brazil, Bahia, Lençóis & $12^{\circ} 33^{\prime} \mathrm{S}, 41^{\circ} 22^{\prime} \mathrm{W}$ & 26 \\
\hline M. adenocarpa & Batocaulon & Simon, M.F. 728 & $\begin{array}{l}\text { Brazil, Distrito Federal, } \\
\text { Brasília }\end{array}$ & $15^{\circ} 45^{\prime} \mathrm{S}, 47^{\circ} 51^{\prime} \mathrm{W}$ & 26 \\
\hline $\begin{array}{l}\text { M. aff. bathyrrhena } \\
\text { Barneby }^{\mathrm{a}}\end{array}$ & Calothamnos & Simon, M.F. 874 & Brazil & & 52 \\
\hline M. affinis B.L.Rob. ${ }^{\mathrm{a}}$ & Mimosa & Martinez-Romero, E. s/n & Mexico & & 26 \\
\hline $\begin{array}{l}\text { M. albida Humb. \& } \\
\text { Bonpl. ex Willd. var. } \\
\text { albida }\end{array}$ & Mimosa & Way, M.J. 158 & Mexico, Yucatan, Opichen & $20^{\circ} 33^{\prime} \mathrm{N}, 89^{\circ} 53^{\prime} \mathrm{W}$ & 26 \\
\hline M. albolanata Taub. $^{\text {a }}$ & Habbasia & Simon, M.F. 677 & Brazil, Goiás, Pirenopolis & $15^{\circ} 47^{\prime} \mathrm{S}, 48^{\circ} 53^{\prime} \mathrm{W}$ & 26 \\
\hline M. antrorsa Benth. ${ }^{\mathrm{a}}$ & Habbasia & Fagg, C.W. 1747 & $\begin{array}{l}\text { Brazil, Distrito Federal, } \\
\text { Brasília }\end{array}$ & $15^{\circ} 51^{\prime} \mathrm{S}, 47^{\circ} 51^{\prime} \mathrm{W}$ & 26 \\
\hline M. apodocarpa Benth. ${ }^{\mathrm{a}}$ & Batocaulon & Simon, M.F.635 & Brazil, Tocantins, Filadélfia & $7^{\circ} 29^{\prime} \mathrm{S}, 47^{\circ} 55^{\prime} \mathrm{W}$ & 26 \\
\hline $\begin{array}{l}\text { M. artemisiana } \\
\text { Heringer \& Paula }^{\mathrm{a}}\end{array}$ & Batocaulon & Faria, S.M. 138 & $\begin{array}{l}\text { Brazil, Minas Gerais, } \\
\text { Marliéria }\end{array}$ & $19^{\circ} 42^{\prime} \mathrm{S}, 42^{\circ} 33^{\prime} \mathrm{W}$ & 26 \\
\hline M. bahamensis Benth. ${ }^{\mathrm{a}}$ & Batocaulon & Way, M.J. 100 & Mexico, Yucatan, Celestún & $20^{\circ} 51^{\prime} \mathrm{N}, 90^{\circ} 07^{\prime} \mathrm{W}$ & 26 \\
\hline M. benthamii Macbride ${ }^{\mathrm{a}}$ & Batocaulon & James, E.K. 335 & $\begin{array}{l}\text { Mexico, Morelos, Sierra de } \\
\text { Huautla }\end{array}$ & $18^{\circ} 29^{\prime} \mathrm{N}, 99^{\circ} 00^{\prime} \mathrm{W}$ & 26 \\
\hline $\begin{array}{l}\text { M. bimucronata Kuntze } \\
\text { var. bimucronata }\end{array}$ & Batocaulon & Simon, M.F. 301 & $\begin{array}{l}\text { Brazil, Distrito Federal, } \\
\text { Brasília }\end{array}$ & $15^{\circ} 43^{\prime} \mathrm{S}, 47^{\circ} 57^{\prime} \mathrm{W}$ & 26 \\
\hline M. bimucronata & Batocaulon & Dahmer, N. 46 & $\begin{array}{l}\text { Brazil, São Paulo, Mogi das } \\
\text { Cruzes }\end{array}$ & $23^{\circ} 30^{\prime} \mathrm{S}, 46^{\circ} 10^{\prime} \mathrm{W}$ & 26 \\
\hline M. bimucronata & Batocaulon & Dahmer, N. 48 & $\begin{array}{l}\text { Brazil, São Paulo, Mogi das } \\
\text { Cruzes }\end{array}$ & $23^{\circ} 33^{\prime} \mathrm{S}, 46^{\circ} 08^{\prime} \mathrm{W}$ & 26 \\
\hline M. biuncifera Benth. ${ }^{\mathrm{a}}$ & Batocaulon & Newman, M. 284 & USA, Arizona & $31^{\circ} 43^{\prime} \mathrm{N}, 111^{\circ} 09^{\prime} \mathrm{W}$ & 52 \\
\hline M. blanchetii Benth. ${ }^{\mathrm{a}}$ & Batocaulon & Coradin, L. 6204 & Brazil, Bahia & $11^{\circ} 27^{\prime} \mathrm{S}, 41^{\circ} 07^{\prime} \mathrm{W}$ & 26 \\
\hline M. borealis A.Gray ${ }^{\mathrm{a}}$ & Batocaulon & Simon, M.F. 873 & USA, cultivated in Oxford & & 26 \\
\hline $\begin{array}{l}\text { M. caesalpiniifolia } \\
\text { Benth. }\end{array}$ & Batocaulon & Dahmer, N. 50 & Brazil, São Paulo, Arujá & $23^{\circ} 29^{\prime} \mathrm{S}, 46^{\circ} 15^{\prime} \mathrm{W}$ & 26 \\
\hline M. caesalpiniifolia & Batocaulon & Dahmer, N. 35 & Brazil, Goiás, Goiânia & $16^{\circ} 40^{\prime} \mathrm{S}, 49^{\circ} 18^{\prime} \mathrm{W}$ & 26 \\
\hline M. caesalpiniifolia & Batocaulon & Dahmer, N. 29 & $\begin{array}{l}\text { Brazil, São Paulo, Mogi das } \\
\text { Cruzes }\end{array}$ & $23^{\circ} 31^{\prime} \mathrm{S}, 46^{\circ} 13^{\prime} \mathrm{W}$ & 26 \\
\hline M. caesalpiniifolia & Batocaulon & Kamiski, P. s/n & Brazil, Roraima & & 26 \\
\hline $\begin{array}{l}\text { M. campicola Harms } \\
\text { var. planipes Barneby }\end{array}$ & Batocaulon & Simon, M.F. 692 & $\begin{array}{l}\text { Brazil, Bahia, Morro do } \\
\text { Chapéu }\end{array}$ & $11^{\circ} 29^{\prime} \mathrm{S}, 41^{\circ} 19^{\prime} \mathrm{W}$ & 26 \\
\hline M. camporum Benth. ${ }^{\mathrm{a}}$ & Habbasia & Sanou, L. BUR-315 & $\begin{array}{l}\text { Burkina Faso, Houet, } \\
\text { Kokozowe }\end{array}$ & $11^{\circ} 08^{\prime} \mathrm{N}, 4^{\circ} 21^{\prime} \mathrm{W}$ & 26 \\
\hline M. camporum & Habbasia & Bako, B.BUR-282 & $\begin{array}{c}\text { Burkina Faso, Comoe, } \\
\text { Yendéré/Niangoloko }\end{array}$ & $10^{\circ} 12^{\prime} \mathrm{N}, 4^{\circ} 59^{\prime} \mathrm{W}$ & 26 \\
\hline M. camporum & Habbasia & Faria, S.M. 729 & $\begin{array}{l}\text { Brazil, Pará, Porto } \\
\text { Trombetas }\end{array}$ & $1^{\circ} 40^{\prime} \mathrm{S}, 56^{\circ} 27^{\prime} \mathrm{W}$ & 26 \\
\hline M. candollei R.Grether ${ }^{\mathrm{a}}$ & Batocaulon & Simon MF 644 & Brazil, Tocantins, Filadélfia & & 52 \\
\hline $\begin{array}{l}\text { M. cisparanensis } \\
\text { Barneby }^{\mathrm{a}}\end{array}$ & Habbasia & Simon, M.F. 568 & $\begin{array}{l}\text { Brazil, Mato Grosso, } \\
\text { Nobres }\end{array}$ & $14^{\circ} 19^{\prime} \mathrm{S}, 55^{\circ} 24^{\prime} \mathrm{W}$ & 52 \\
\hline M. claussenii Benth. $^{\mathrm{a}}$ & Habbasia & Simon, M.F. 303 & Brazil, Distrito Federal & $15^{\circ} 43^{\prime} \mathrm{S}, 47^{\circ} 57^{\prime} \mathrm{W}$ & 26 \\
\hline M. claussenii & Habbasia & Simon, M.F. 308 & Brazil, Goiás & $15^{\circ} 58^{\prime} \mathrm{S}, 48^{\circ} 50^{\prime} \mathrm{W}$ & 26 \\
\hline
\end{tabular}


Table 1 continued

\begin{tabular}{|c|c|c|c|c|c|}
\hline Species & Section & Accession & Place of collection & Coordinates & Chromosome no. \\
\hline M. cordistipula Benth. ${ }^{\mathrm{a}}$ & Batocaulon & Simon, M.F. 693 & $\begin{array}{l}\text { Brazil, Bahia, Morro do } \\
\text { Chapéu }\end{array}$ & $11^{\circ} 29^{\prime} \mathrm{S}, 41^{\circ} 19^{\prime} \mathrm{W}$ & 26 \\
\hline $\begin{array}{l}\text { M. cryptothamnos } \\
\text { Barneby }^{\mathrm{a}}\end{array}$ & Habbasia & Simon, M.F. 738 & $\begin{array}{l}\text { Brazil, Goiás, São João da } \\
\text { Aliança }\end{array}$ & $14^{\circ} 52^{\prime} \mathrm{S}, 47^{\circ} 34^{\prime} \mathrm{W}$ & 26 \\
\hline $\begin{array}{l}\text { M. debilis Humb. \& } \\
\text { Bonpl. ex Willd. var. } \\
\text { debilis }\end{array}$ & Mimosa & Simon, M.F. 778 & Brazil, Mato Grosso & $15^{\circ} 50^{\prime} \mathrm{S}, 56^{\circ} 06^{\prime} \mathrm{W}$ & 26 \\
\hline M. debilis & Mimosa & Simon, M.F. 790 & $\begin{array}{l}\text { Brazil, Mato Grosso, } \\
\text { Chapada dos Guimarães }\end{array}$ & $15^{\circ} 20^{\prime} \mathrm{S}, 55^{\circ} 54^{\prime} \mathrm{W}$ & 26 \\
\hline $\begin{array}{l}\text { M. debilis var. vestita } \\
\text { (Benth.) Barneby }\end{array}$ & Mimosa & Simon, M.F. 783 & Brazil, Mato Grosso & $15^{\circ} 50^{\prime} \mathrm{S}, 56^{\circ} 05^{\prime} \mathrm{W}$ & 26 \\
\hline M. delicatula Baill. $^{\mathrm{a}}$ & Batocaulon & Sutherland, J.M. 262 & Madagascar, Amborosary & $25^{\circ} 01^{\prime} \mathrm{S}, 46^{\circ} 38^{\prime} \mathrm{E}$ & 26 \\
\hline M. depauperata Benth. ${ }^{\mathrm{a}}$ & Batocaulon & Simon, M.F. 801 & $\begin{array}{l}\text { Mexico, Queretaro, } \\
\text { Tequisquiapan }\end{array}$ & $20^{\circ} 33^{\prime} \mathrm{N}, 99^{\circ} 54^{\prime} \mathrm{W}$ & 26 \\
\hline $\begin{array}{l}\text { M. diplotricha C.Wright } \\
\text { ex Sauvalle var. } \\
\text { diplotricha }\end{array}$ & Batocaulon & Simon, M.F. 304 & $\begin{array}{l}\text { Brazil, Distrito Federal, } \\
\text { Lago Sul }\end{array}$ & $15^{\circ} 52^{\prime} \mathrm{S}, 47^{\circ} 50^{\prime} \mathrm{W}$ & 26 \\
\hline $\begin{array}{l}\text { M. diplotricha } \mathrm{C} \text {. } \\
\text { Wright ex Sauvalle }\end{array}$ & Batocaulon & Simon, M.F. 877 & Taiwan, Ping-Tung & $22^{\circ} 45^{\prime} \mathrm{N}, 120^{\circ} 34^{\prime} \mathrm{E}$ & 26 \\
\hline $\begin{array}{l}\text { M. dominarum } \\
\text { Barneby }^{\mathrm{a}}\end{array}$ & Habbasia & Simon, M.F. 776 & Brazil, Goiás, Alto Paraíso & $14^{\circ} 11^{\prime} \mathrm{S}, 47^{\circ} 29^{\prime} \mathrm{W}$ & 26 \\
\hline M. dysocarpa Benth. ${ }^{\mathrm{a}}$ & Batocaulon & Newman, M. 296 & USA, Arizona & $31^{\circ} 47^{\prime} \mathrm{N}, 110^{\circ} 49^{\prime} \mathrm{W}$ & 26 \\
\hline M. echinocaula Benth. ${ }^{a}$ & Batocaulon & Simon, M.F. 312 & $\begin{array}{l}\text { Brazil, Goiás, Formosa- } \\
\text { Itiquira }\end{array}$ & $15^{\circ} 32^{\prime} \mathrm{S}, 47^{\circ} 25^{\prime} \mathrm{W}$ & 26 \\
\hline M. flocculosa Burk. $^{\mathrm{a}}$ & Calothamnos & CNPF $s / n$ & Brazil, Paraná & & 26 \\
\hline M. foliolosa Benth. $^{\mathrm{a}}$ & Habbasia & Simon, M.F. 321 & $\begin{array}{l}\text { Brazil, Distrito Federal, } \\
\text { Brasília }\end{array}$ & $15^{\circ} 43^{\prime} \mathrm{S}, 47^{\circ} 57^{\prime} \mathrm{W}$ & 26 \\
\hline M. foliolosa & Habbasia & Simon, M.F. 663 & $\begin{array}{l}\text { Brazil, Distrito Federal, } \\
\text { Planaltina }\end{array}$ & $15^{\circ} 36^{\prime} \mathrm{S}, 47^{\circ} 44^{\prime} \mathrm{W}$ & 26 \\
\hline M. foliolosa & Habbasia & Simon, M.F.672 & Brazil, Goiás, Pirenopolis & $15^{\circ} 46^{\prime} \mathrm{S}, 48^{\circ} 56^{\prime} \mathrm{W}$ & 26 \\
\hline $\begin{array}{l}\text { M. goldmanii B. } \\
\text { L. Rob. }^{\text {a }}\end{array}$ & Mimosa & James, EK 326 & $\begin{array}{l}\text { México, Morelos, Sierra de } \\
\text { Huautla }\end{array}$ & $18^{\circ} 28^{\prime} \mathrm{N}, 99^{\circ} 01^{\prime} \mathrm{W}$ & 52 \\
\hline M. gracilis Benth. $^{\mathrm{a}}$ & Batocaulon & Simon, M.F. 323 & $\begin{array}{l}\text { Brazil, Minas Gerais, PN } \\
\text { Sertão Veredas }\end{array}$ & $15^{\circ} 23^{\prime} \mathrm{S}, 45^{\circ} 54^{\prime} \mathrm{W}$ & 26 \\
\hline M. hamata Willd. & Batocaulon & Simon, M.F. 876 & Índia & & 26 \\
\hline M. heringeri Barneby ${ }^{\mathrm{a}}$ & Habbasia & Proença, C.2138 & $\begin{array}{l}\text { Brazil, Distrito Federal, } \\
\text { Gama }\end{array}$ & $16^{\circ} 03^{\prime} \mathrm{S}, 48^{\circ} 03^{\prime} \mathrm{W}$ & 26 \\
\hline M. hexandra Micheli $^{\mathrm{a}}$ & Batocaulon & Way, M.J. 2440 & Brazil, Bahia & $9^{\circ} 20^{\prime} \mathrm{S}, 40^{\circ} 18^{\prime} \mathrm{W}$ & 26 \\
\hline $\begin{array}{l}\text { M. hypoglauca Mart. } \\
\text { var. hypoglauca }\end{array}$ & Mimosa & Simon, M.F. 723 & Brazil, Bahia, Palmeiras & $12^{\circ} 45^{\prime} \mathrm{S}, 41^{\circ} 30^{\prime} \mathrm{W}$ & 26 \\
\hline $\begin{array}{l}\text { M. incana (Spreng.) } \\
\text { Benth. }^{\mathrm{a}}\end{array}$ & Calothamnos & Dahmer, N. 1 & $\begin{array}{l}\text { Brazil, Rio Grande do Sul, } \\
\text { Porto Alegre }\end{array}$ & $29^{\circ} 53^{\prime} \mathrm{S}, 50^{\circ} 18^{\prime} \mathrm{W}$ & 52 \\
\hline M. incana & Calothamnos & Dahmer, N. 2 & $\begin{array}{l}\text { Brazil, Rio Grande do Sul, } \\
\text { Tainhas }\end{array}$ & $29^{\circ} 25^{\prime} \mathrm{S}, 50^{\circ} 30^{\prime} \mathrm{W}$ & 52 \\
\hline M. incana & Calothamnos & Dahmer, N. 17 & $\begin{array}{l}\text { Brazil, Rio Grande do Sul, } \\
\text { Caxias do Sul }\end{array}$ & $29^{\circ} 04^{\prime} \mathrm{S}, 51^{\circ} 00^{\prime} \mathrm{W}$ & 52 \\
\hline M. incana & Calothamnos & Dahmer, N. 22 & $\begin{array}{l}\text { Brazil, Rio Grande do Sul, } \\
\text { Caxias do Sul }\end{array}$ & $29^{\circ} 15^{\prime} \mathrm{S}, 50^{\circ} 20^{\prime} \mathrm{W}$ & 52 \\
\hline M. incana & Calothamnos & Dahmer, N. 26 & $\begin{array}{l}\text { Brazil, Rio Grande do Sul, } \\
\text { São Francisco de Paula }\end{array}$ & $29^{\circ} 25^{\prime} \mathrm{S}, 50^{\circ} 30^{\prime} \mathrm{W}$ & 52 \\
\hline $\begin{array}{l}\text { M. invisa Mart. ex Colla } \\
\text { var. invisa }{ }^{\text {a }}\end{array}$ & Batocaulon & Feltwell, J. 19 & Indonesia, Sulawesi & $0^{\circ} 33^{\prime} \mathrm{N}, 123^{\circ} 54^{\prime} \mathrm{E}$ & 26 \\
\hline M. lacerata Rose $^{\mathrm{a}}$ & Batocaulon & Simon, M.F. 806 & $\begin{array}{l}\text { Mexico, Queretaro, } \\
\text { Toliman }\end{array}$ & $20^{\circ} 51^{\prime} \mathrm{N}, 99^{\circ} 52^{\prime} \mathrm{W}$ & 26 \\
\hline
\end{tabular}


Table 1 continued

\begin{tabular}{|c|c|c|c|c|c|}
\hline Species & Section & Accession & Place of collection & Coordinates & Chromosome no. \\
\hline M. latispinosa Lam. $^{\mathrm{a}}$ & Batocaulon & Sutherland, J.M. 206 & Madagascar, Tana & $18^{\circ} 54^{\prime} \mathrm{S}, 47^{\circ} 30^{\prime} \mathrm{E}$ & 26 \\
\hline M. luisana Brandegee $^{\mathrm{a}}$ & Batocaulon & Way, M.J. 09 & $\begin{array}{l}\text { Mexico, Puebla, San Jose } \\
\text { Axuxco }\end{array}$ & $18^{\circ} 11^{\prime} \mathrm{N}, 97^{\circ} 11^{\prime} \mathrm{W}$ & 26 \\
\hline M. melanocarpa Benth. $^{\text {a }}$ & Habbasia & Simon, M.F. 675 & Brazil, Goiás, Pirenópolis & $15^{\circ} 46^{\prime} \mathrm{S}, 49^{\circ} 00^{\prime} \mathrm{W}$ & 26 \\
\hline M. misera Benth. $^{\mathrm{a}}$ & Batocaulon & Simon, M.F. 703 & Brazil, Bahia, Xique-Xique & $10^{\circ} 57^{\prime} \mathrm{S}, 42^{\circ} 43^{\prime} \mathrm{W}$ & 26 \\
\hline M. monancistra Benth. $^{\mathrm{a}}$ & Batocaulon & Simon, M.F. 809 & $\begin{array}{l}\text { Mexico, Guanajuato, San } \\
\text { Miguel Allende }\end{array}$ & $21^{\circ} 01^{\prime} \mathrm{N}, 100^{\circ} 47^{\prime} \mathrm{W}$ & 26 \\
\hline M. neptuniodes Harms ${ }^{\mathrm{a}}$ & Habbasia & Wood, J.R.I. 22123 & Bolivia, Santa Cruz & $17^{\circ} 33^{\prime} \mathrm{S}, 63^{\circ} 21^{\prime} \mathrm{W}$ & 26 \\
\hline M. niederleinii Burk. ${ }^{\mathrm{a}}$ & Mimosa & Dahmer, N. 23 & $\begin{array}{l}\text { Brazil, Rio Grande do Sul, } \\
\text { Tainhas }\end{array}$ & $29^{\circ} 16^{\prime} \mathrm{S}, 50^{\circ} 19^{\prime} \mathrm{W}$ & 52 \\
\hline $\begin{array}{l}\text { M. nuda Benth. var. } \\
\text { nuda }^{\mathrm{a}}\end{array}$ & Mimosa & Simon, M.F. 793 & $\begin{array}{l}\text { Brazil, Mato Grosso, } \\
\text { Chapada dos Guimarães }\end{array}$ & $15^{\circ} 20^{\prime} \mathrm{S}, 56^{\circ} 46^{\prime} \mathrm{W}$ & 52 \\
\hline $\begin{array}{l}\text { M. nuttallii (DC.) } \\
\text { B.L.Turner }\end{array}$ & Batocaulon & Simon, M.F. 875 & USA, cultivated in Oxford & & 26 \\
\hline M. nuttallii & Batocaulon & Allen, B. CBG-05-100 & USA, Kansas & $39^{\circ} 06^{\prime} \mathrm{N}, 96^{\circ} 36^{\prime} \mathrm{W}$ & 26 \\
\hline $\begin{array}{l}\text { M. ophthalmocentra } \\
\text { Mart. Ex Benth. }{ }^{\mathrm{a}}\end{array}$ & Batocaulon & Way, M.J. 2434 & $\begin{array}{l}\text { Brazil, Pernambuco, } \\
\text { Petrolina }\end{array}$ & $9^{\circ} 02^{\prime} \mathrm{S}, 40^{\circ} 38^{\prime} \mathrm{W}$ & 26 \\
\hline $\begin{array}{l}\text { M. orthocarpa Spruce } \\
\text { Ex Benth. }^{\text {a }}\end{array}$ & Batocaulon & Grether, R. 2907 & $\begin{array}{l}\text { Mexico, Veracruz, Las } \\
\text { Choapas }\end{array}$ & $18^{\circ} 01^{\prime} \mathrm{N}, 95^{\circ} 08^{\prime} \mathrm{W}$ & 26 \\
\hline M. orthocarpa & Batocaulon & Simon, M.F. 855 & $\begin{array}{l}\text { Mexico, Veracruz, } \\
\text { Acayucan }\end{array}$ & $17^{\circ} 58^{\prime} \mathrm{N}, 95^{\circ} 11^{\prime} \mathrm{W}$ & 26 \\
\hline $\begin{array}{l}\text { M. papposa Benth. var. } \\
\text { papposa }^{\mathrm{a}}\end{array}$ & Mimosa & Proença, C 3431 & Brazil, Distrito Federal & $15^{\circ} 35^{\prime} \mathrm{S}, 48^{\circ} 04^{\prime} \mathrm{W}$ & 26 \\
\hline $\begin{array}{l}\text { M. pigra L. var. } \\
\text { dehiscens (Barneby) } \\
\text { D.Glazier \& } \\
\text { Mackinder }\end{array}$ & Habbasia & Dahmer, N. 28 & $\begin{array}{l}\text { Brazil, São Paulo, Mogi das } \\
\text { Cruzes }\end{array}$ & $23^{\circ} 31^{\prime} \mathrm{S}, 6^{\circ} 13^{\prime} \mathrm{W}$ & 52 \\
\hline M. pigra var. dehiscens & Habbasia & Dahmer, N. 32 & Brazil, Goiás, Caldas Novas & $17^{\circ} 44^{\prime} \mathrm{S}, 48^{\circ} 38^{\prime} \mathrm{W}$ & 52 \\
\hline M. pigra var. dehiscens & Habbasia & Sarkinen, T.E. 2059 & $\begin{array}{l}\text { Bolivia, La Paz, Sud } \\
\text { Yungas }\end{array}$ & $16^{\circ} 23^{\prime} \mathrm{S}, 67^{\circ} 31^{\prime} \mathrm{W}$ & 26 \\
\hline M. pigra var. dehiscens & Habbasia & Simon, M.F. s.n. & Brazil, Goiás, Cristalina & $16^{\circ} 46^{\prime} \mathrm{S}, 47^{\circ} 36^{\prime} \mathrm{W}$ & 26 \\
\hline M. pigra var. dehiscens & Habbasia & Simon, M.F. 799 & $\begin{array}{l}\text { Brazil, Mato Grosso, } \\
\text { Pocone }\end{array}$ & $16^{\circ} 30^{\prime} \mathrm{S}, 56^{\circ} 42^{\prime} \mathrm{W}$ & 52 \\
\hline M. polyantha Benth. ${ }^{\mathrm{a}}$ & Batocaulon & Simon, M.F. 827 & Mexico, Sinaloa, Rosario & $22^{\circ} 53^{\prime} \mathrm{N}, 105^{\circ} 48^{\prime} \mathrm{W}$ & 26 \\
\hline M. polyantha & Batocaulon & Way, M.J. WSO21 & $\begin{array}{l}\text { Mexico, Oaxaca, San Juan } \\
\text { de los Cues }\end{array}$ & $18^{\circ} 02^{\prime} \mathrm{N}, 97^{\circ} 03^{\prime} \mathrm{W}$ & 26 \\
\hline $\begin{array}{l}\text { M. polycarpa Kunth } \\
\text { var. subandina } \\
\text { Barneby }^{\mathrm{a}}\end{array}$ & Mimosa & Sarkinen, T.E. 2061 & $\begin{array}{l}\text { Bolivia, La Paz, Sud } \\
\text { Yungas }\end{array}$ & $16^{\circ} 23^{\prime} \mathrm{S}, 67^{\circ} 31^{\prime} \mathrm{W}$ & 26 \\
\hline $\begin{array}{l}\text { M. polydactyla Humb. } \\
\text { \& Bonpl. ex Willd. }\end{array}$ & Mimosa & Thomas, S.M. 30/3 & Ecuador, Napo, Coca & & 26 \\
\hline M. polydactyla & Mimosa & Coradin, L. 8682 & Brazil, Bahia, Ilhéus & $14^{\circ} 44^{\prime} \mathrm{S}, 39^{\circ} 09^{\prime} \mathrm{W}$ & 26 \\
\hline $\begin{array}{l}\text { M. polydidyma } \\
\text { Barneby }^{\mathrm{a}}\end{array}$ & Batocaulon & Simon, M.F. 719 & Brazil, Bahia, Palmeiras & $12^{\circ} 38^{\prime} \mathrm{S}, 41^{\circ} 33^{\prime} \mathrm{W}$ & 26 \\
\hline M. pteridifolia Benth. $^{\mathrm{a}}$ & Batocaulon & Simon, M.F. 311 & Brazil, Goiás, Formosa & $15^{\circ} 32^{\prime} \mathrm{S}, 47^{\circ} 25^{\prime} \mathrm{W}$ & 26 \\
\hline M. pudica $\mathrm{L}$. & Mimosa & MSB 19956 & & & 52 \\
\hline $\begin{array}{l}\text { M. pudica } \text { L. var. } \\
\text { hispida } \text { Brenan }^{\mathrm{a}}\end{array}$ & Mimosa & Simon, M.F. 893 & Brazil, Distrito Federal & $15^{\circ} 44^{\prime} \mathrm{S}, 47^{\circ} 52^{\prime} \mathrm{W}$ & 52 \\
\hline M. pudica & Mimosa & Beer, L. 394 & Nepal & & 52 \\
\hline M. pycnocoma Benth. $^{\mathrm{a}}$ & Habbasia & Simon, M.F. 868 & Brazil, Goiás, Cavalcante & $13^{\circ} 32^{\prime} \mathrm{S}, 47^{\circ} 29^{\prime} \mathrm{W}$ & 52 \\
\hline M. radula Benth. $^{\mathrm{a}}$ & Mimosa & Simon, M.F. 296 & Brazil, Goiás, Pirenopolis & $15^{\circ} 48^{\prime} \mathrm{S}, 48^{\circ} 50^{\prime} \mathrm{W}$ & 26 \\
\hline M. revoluta Benth. $^{a}$ & Mimadenia & Sarkinen, T.E. 2074 & Bolivia, La Paz, Inquiisivi & $16^{\circ} 41^{\prime} \mathrm{S}, 67^{\circ} 14^{\prime} \mathrm{W}$ & 26 \\
\hline
\end{tabular}


Table 1 continued

\begin{tabular}{|c|c|c|c|c|c|}
\hline Species & Section & Accession & Place of collection & Coordinates & Chromosome no. \\
\hline M. robusta $\mathrm{R}$. Grether ${ }^{\mathrm{a}}$ & Batocaulon & Simon, M.F. 863 & $\begin{array}{l}\text { Mexico, Jalisco, Puerto } \\
\text { Vallarta }\end{array}$ & $20^{\circ} 29^{\prime} \mathrm{N}, 105^{\circ} 18^{\prime} \mathrm{W}$ & 52 \\
\hline $\begin{array}{l}\text { M. rubicaulis Lam. ssp. } \\
\text { himalayana (Gamble) } \\
\text { H. Ohashi }^{\text {a }}\end{array}$ & Batocaulon & Thomas, S.M. 24/1 & Nepal & & 26 \\
\hline M. scabrella Benth. ${ }^{a}$ & Calothamnos & Dahmer, N. 14 & $\begin{array}{l}\text { Brazil, Rio Grande do Sul, } \\
\text { Caxias do Sul }\end{array}$ & $29^{\circ} 08^{\prime} \mathrm{S}, 51^{\circ} 05^{\prime} \mathrm{W}$ & 52 \\
\hline M. scabrella & Calothamnos & Lima, H.C. 4055 & $\begin{array}{l}\text { Brazil, Minas Gerais, Ouro } \\
\text { Preto }\end{array}$ & $20^{\circ} 17^{\prime} \mathrm{N}, 45^{\circ} 30^{\prime} \mathrm{W}$ & 52 \\
\hline $\begin{array}{l}\text { M. schomburgkii } \\
\text { Benth. }^{\mathrm{a}}\end{array}$ & Batocaulon & Hellin, J.J. 15 & Honduras, Colon & $15^{\circ} 45^{\prime} \mathrm{N}, 85^{\circ} 44^{\prime} \mathrm{W}$ & 26 \\
\hline $\begin{array}{l}\text { M. sensitiva } \mathrm{L} \text {. var. } \\
\text { sensitiva }^{\text {a }}\end{array}$ & Mimosa & Conceição, S.F. 284 & Brazil, Bahia & & 26 \\
\hline M. sericantha Benth. ${ }^{\mathrm{a}}$ & Batocaulon & Simon, M.F. 410 & Brazil, Tocantins & $10^{\circ} 26^{\prime} \mathrm{S}, 46^{\circ} 27^{\prime} \mathrm{W}$ & 26 \\
\hline $\begin{array}{l}\text { M. setosa } \text { Benth. var. } \\
\text { paludosa (Benth.) } \\
\text { Barneby }^{\mathrm{a}}\end{array}$ & Habbasia & Simon, M.F. 306 & Brazil, Distrito Federal & $15^{\circ} 45^{\prime} \mathrm{S}, 47^{\circ} 51^{\prime} \mathrm{W}$ & 26 \\
\hline M. setosa var. paludosa & Habbasia & Simon, M.F. 666 & $\begin{array}{l}\text { Brazil, Distrito Federal, } \\
\text { Brasília }\end{array}$ & $15^{\circ} 45^{\prime} \mathrm{S}, 47^{\circ} 51^{\prime} \mathrm{W}$ & 52 \\
\hline M. setosa var. paludosa & Habbasia & Dahmer, N. 27 & $\begin{array}{l}\text { Brazil, São Paulo, Mogi das } \\
\text { Cruzes }\end{array}$ & $23^{\circ} 32^{\prime} \mathrm{S}, 46^{\circ} 12^{\prime} \mathrm{W}$ & 52 \\
\hline M. setosa var. paludosa & Habbasia & Dahmer, N. 49 & $\begin{array}{l}\text { Brazil, São Paulo, Mogi das } \\
\text { Cruzes }\end{array}$ & $23^{\circ} 28^{\prime} \mathrm{S}, 46^{\circ} 14^{\prime} \mathrm{W}$ & 52 \\
\hline $\begin{array}{l}\text { M. setosa Benth. var. } \\
\text { urbica } \text { Barneby }^{\mathrm{a}}\end{array}$ & Habbasia & Simon, M.F. 730 & $\begin{array}{l}\text { Brazil, Distrito Federal, } \\
\text { Brasília }\end{array}$ & $15^{\circ} 45^{\prime} \mathrm{S}, 47^{\circ} 51^{\prime} \mathrm{W}$ & 26 \\
\hline M. setosissima Taub. ${ }^{\mathrm{a}}$ & Habbasia & Simon, M.F. 290 & Brazil, Goiás, Cocalzinho & $15^{\circ} 48^{\prime} \mathrm{S}, 48^{\circ} 47^{\prime} \mathrm{W}$ & 26 \\
\hline $\begin{array}{l}\text { M. similis Britton \& } \\
\text { Rose }^{\mathrm{a}}\end{array}$ & Batocaulon & James, E.K. 229 & $\begin{array}{l}\text { Mexico, Queretaro, } \\
\text { Cadereyta }\end{array}$ & $20^{\circ} 55^{\prime} \mathrm{N}, 99^{\circ} 45^{\prime} \mathrm{W}$ & 26 \\
\hline M. similis & Batocaulon & Simon, M.F. 807 & $\begin{array}{l}\text { Mexico, Queretaro, } \\
\text { Cadereyta }\end{array}$ & $20^{\circ} 56^{\prime} \mathrm{N}, 99^{\circ} 44^{\prime} \mathrm{W}$ & 26 \\
\hline $\begin{array}{l}\text { M. skinneri Benth. var. } \\
\text { skinneri }^{\text {a }}\end{array}$ & Mimosa & James, E.K. 244 & Mexico, Jalisco, Tepic & $21^{\circ} 14^{\prime} \mathrm{N}, 104^{\circ} 38^{\prime} \mathrm{W}$ & 52 \\
\hline $\begin{array}{l}\text { M. somnians Humb. \& } \\
\text { Bonpl. ex Willd. }\end{array}$ & Habbasia & Grether, R. 2901 & Mexico, Jalisco & $21^{\circ} 18^{\prime} \mathrm{N}, 104^{\circ} 39^{\prime} \mathrm{W}$ & 52 \\
\hline M. somnians & Habbasia & Simon, M.F. 797 & $\begin{array}{l}\text { Brazil, Mato Grosso, } \\
\text { Poconé }\end{array}$ & $16^{\circ} 20^{\prime} \mathrm{S}, 56^{\circ} 38^{\prime} \mathrm{W}$ & 26 \\
\hline M. somnians & Habbasia & Way, M.J. 2476 & $\begin{array}{l}\text { Brazil, Rio Grande do } \\
\text { Norte }\end{array}$ & $4^{\circ} 53^{\prime} \mathrm{S}, 37^{\circ} 17^{\prime} \mathrm{W}$ & 52 \\
\hline $\begin{array}{l}\text { M. somnians var. } \\
\text { viscida (Willd.) } \\
\text { Barneby }\end{array}$ & Habbasia & Simon, M.F. 863 & Brazil, Goiás, Alto Paraíso & $14^{\circ} 05^{\prime} \mathrm{S}, 47^{\circ} 30^{\prime} \mathrm{W}$ & 26 \\
\hline $\begin{array}{l}\text { M. sparsiformis } \\
\text { Barneby }^{\mathrm{a}}\end{array}$ & Mimosa & Dahmer, N. 15 & $\begin{array}{l}\text { Brazil, Rio Grande do Sul, } \\
\text { Caxias do Sul }\end{array}$ & $29^{\circ} 08^{\prime} \mathrm{S}, 51^{\circ} 03^{\prime} \mathrm{W}$ & 39 \\
\hline M. sparsiformis & Mimosa & Dahmer, N. 52 & $\begin{array}{l}\text { Brazil, Rio Grande do Sul, } \\
\text { Caxias do Sul }\end{array}$ & $29^{\circ} 12^{\prime} \mathrm{S}, 51^{\circ} 17^{\prime} \mathrm{W}$ & 39 \\
\hline M. splendida Barneby $^{\mathrm{a}}$ & Habbasia & Simon, M.F. 316 & Brazil, Goiás, C. Veadeiros & $14^{\circ} 10^{\prime} \mathrm{S}, 47^{\circ} 35^{\prime} \mathrm{W}$ & 26 \\
\hline $\begin{array}{l}\text { M. tenuiflora (Willd.) } \\
\text { Poir. }^{\mathrm{a}}\end{array}$ & Batocaulon & Camargo-Ricalde, S.L. 98 & Mexico, Oaxaca, Zanatepec & $16^{\circ} 26^{\prime} \mathrm{N}, 95^{\circ} 01^{\prime} \mathrm{W}$ & 26 \\
\hline M. tenuiflora & Batocaulon & Way, M.J. 2441 & Brazil, Bahia & $9^{\circ} 20^{\prime} \mathrm{S}, 40^{\circ} 13^{\prime} \mathrm{W}$ & 26 \\
\hline M. ulei Taub. var. $u l e i^{\mathrm{a}}$ & Habbasia & Simon, M.F. 758 & Brazil, Goiás, Alto Paraíso & $14^{\circ} 09^{\prime} \mathrm{S}, 47^{\circ} 37^{\prime} \mathrm{W}$ & 26 \\
\hline $\begin{array}{l}\text { M. uraguensis Hook. \& } \\
\text { Arn. }\end{array}$ & Batocaulon & Simon, M.F.862 & $\begin{array}{l}\text { Uruguay, cultivated in } \\
\text { Oxford }\end{array}$ & & 26 \\
\hline M. ursina Mart. ${ }^{\mathrm{a}}$ & Miтоsa & Simon, M.F. 704 & Brazil, Bahia, Xique-Xique & $10^{\circ} 57^{\prime} \mathrm{S}, 42^{\circ} 43^{\prime} \mathrm{W}$ & 26 \\
\hline
\end{tabular}


Table 1 continued

\begin{tabular}{|c|c|c|c|c|c|}
\hline Species & Section & Accession & Place of collection & Coordinates & Chromosome no. \\
\hline $\begin{array}{l}\text { M. velloziana Mart. var. } \\
\text { velloziana }^{\mathrm{a}}\end{array}$ & Mimosa & Dahmer, N. 45 & $\begin{array}{l}\text { Brazil, São Paulo, Mogi das } \\
\text { Cruzes }\end{array}$ & $23^{\circ} 30^{\prime} \mathrm{S}, 46^{\circ} 10^{\prime} \mathrm{W}$ & 52 \\
\hline $\begin{array}{l}\text { M. velloziana var. } \\
\text { velloziana }\end{array}$ & Mimosa & Simon, M.F. 302 & Brazil, Distrito Federal & $15^{\circ} 43^{\prime} \mathrm{S}, 47^{\circ} 57^{\prime} \mathrm{W}$ & 52 \\
\hline $\begin{array}{l}\text { M. velloziana var. } \\
\text { velloziana }\end{array}$ & Mimosa & Simon, M.F. 665 & $\begin{array}{l}\text { Brazil, Distrito Federal, } \\
\text { Planaltina }\end{array}$ & $15^{\circ} 35^{\prime} \mathrm{S}, 47^{\circ} 42^{\prime} \mathrm{W}$ & 52 \\
\hline M. verrucosa Benth. ${ }^{\mathrm{a}}$ & Batocaulon & Conceição, A.A. 1522 & Brazil, Bahia & $10^{\circ} 00^{\prime} \mathrm{S}, 42^{\circ} 47^{\prime} \mathrm{W}$ & 26 \\
\hline M. virgula Barneby $^{\mathrm{a}}$ & Mimosa & Simon, M.F. 294 & Brazil, Goiás, Pirenópolis & $15^{\circ} 50^{\prime} \mathrm{S}, 48^{\circ} 54^{\prime} \mathrm{W}$ & 26 \\
\hline
\end{tabular}

${ }^{a}$ First determination of chromosome number

at $4^{\circ} \mathrm{C}$, fixed in 6:3:1 (ethanol:chloroform:acetic acid) for $12-24 \mathrm{~h}$ and stored in $70 \%$ alcohol below $0^{\circ} \mathrm{C}$ until required. Slides were prepared by hydrolyzing the roots with $1 \mathrm{~N} \mathrm{HCl}$ at $60^{\circ} \mathrm{C}$ for $8-10 \mathrm{~min}$ and staining with the Feulgen technique for $2-3 \mathrm{~h}$ (sometimes followed by a $2 \%$ pectinase treatment for $2 \mathrm{~min}$ ) and squashed in propionic carmine. At least ten cells with good chromosome spreading and at equivalent contraction were analysed per plant. The best cells were photographed and/or registered by a digital image capturing system.

\section{Results}

Chromosome numbers were determined for the 125 accessions of 92 taxa (from 83 species and one subspecies) of Mimosa (Table 1). For 69 species, 8 varieties and 1 subspecies, chromosome numbers are presented here for the first time, for 6 species and 1 variety previously published counts data have been confirmed, and for 3 species and 2 varieties different numbers were found (Table 1). Results show that $92(74 \%)$ of the accessions analysed are diploid $(2 n=2 x=26)$ (Figs. 1-6) and 33 (26\%) are polyploid, 31 of which are tetraploid $(2 n=4 x=52)$ (Figs. 7, 8) and the 2 accessions of $M$. sparsiformis Barneby are triploid $(2 n=3 x=39)$. This is the first report of a triploid species in the genus. Polysomaty, the occurrence in the root tips of a varying, generally low, percentage of cells with double the normal chromosome number, was reported in some $(26.5 \%)$ of the accessions (data not shown).

Mimosa chromosomes are small, ca. 1-2 $\mu \mathrm{m}$ (Fig. 1). Chromosome measures were not made for all species, but as seen in Fig. 1, there are probably differences in chromosome size among some species and a tendency for tetraploid species to have smaller chromosomes than diploid ones.

Following Barneby's (1991) infrageneric classification, in section Mimadenia, the only species analysed (M. revoluta Benth.) is diploid. This is the first chromosome number determination in this section.
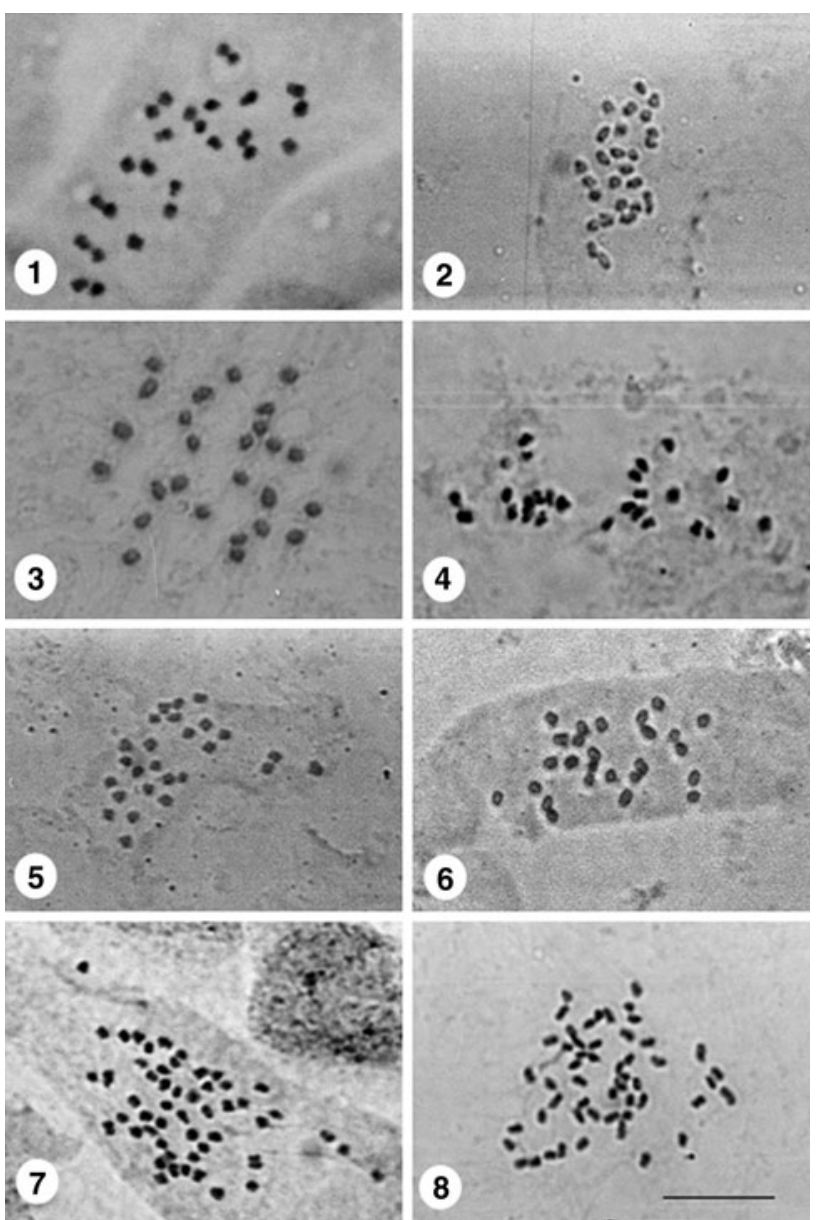

Figs. 1-8 Fig. 1 Mimosa delicatula Sutherland, J.M. $262(2 n=26)$. Fig. $2 M$. rubicaulis ssp. himalayana Thomas, S.M. $24 / 1(2 n=26)$. Fig. 3 M. orthocarpa Grether, R. $2907(2 n=26)$. Fig. 4 M. antrorsa Fagg, C.W. $1747(2 n=26)$. Fig. 5 M. claussenii Simon, M.F. 308 $(2 n=26$.). Fig. 6 M. monancistra Simon, M.F. $809(2 n=26)$. Fig. 7 M. scabrella Dahmer, N. $14(2 n=52)$. Fig. 8 M. robusta Simon, M.F. $863(2 n=52)$

The samples analysed for section Batocaulon (Table 1) are the most representative in terms of geographic range. The 50 accessions and 43 taxa (41 species) analysed were 
collected in several regions of the world, including Brazil (21 taxa), Mexico (10 taxa), U.S.A. (4 taxa), Madagascar (2 taxa), Peru, Taiwan, India, Indonesia, Honduras and Uruguay. Thirty-eight species and one subspecies are diploid [M. acantholoba (Willd.) Poir. var. acantholoba, M. adenocarpa Benth., M. apodocarpa Benth., M. artemisiana Heringer \& Paula, M. bahamensis Benth., M. benthamii Macbride, M. bimucronata Kuntze, M. bimucronata Kuntze var. bimucronata, M. blanchetii Benth., M. borealis A.Gray, M. caesalpiniifolia, M. campicola Harms var. planipes Barneby, M. cordistipula Benth., M. delicatula Baill., M. depauperata Benth., M. diplotricha C.Wright ex Sauvalle var. diplotricha, M. dysocarpa Benth., M. echinocaula Benth., M. gracilis Benth., M. hamata Willd., M. hexandra Micheli, M. invisa Mart. ex Colla var. invisa, M. lacerata Rose, M. latispinosa Lam., M. luisana Brandegee, M. misera Benth., M. monancistra Benth., M. nuttallii (DC.) B.L.Turner, M. ophthalmocentra Mart. ex Benth., M. orthocarpa Spruce ex Benth., M. polyantha Benth., M. polydidyma Barneby, M. pteridifolia Benth., M. rubicaulis Lam. ssp. himalayana (Gamble) H. Ohashi, M. schomburgkii Benth., M. sericantha Benth., M. similis Britton \& Rose, M. tenuiflora, M. uraguensis Hook. \& Arn. and M. verrucosa Benth.] and three are tetraploid [M. biuncifera Benth., M. candollei R. Grether (=M. quadrivalvis L. var. leptocarpa (DC.) Barneby) and $M$. robusta R.Grether].

Nine accessions of four species of section Calothamnos were analysed: one, M. flocculosa Burk., is diploid, and three, M. aff. bathyrrhena Barneby, M. incana (Spreng.) Benth. and M. scabrella, are tetraploid. In section Habbasia 34 accessions of 18 species and 2 varieties were examined: 15 are diploid [M. albolanata Taub., M. antrorsa Benth., M. camporum Benth., M. claussenii Benth., M. cryptothamnos Barneby, M. dominarum Barneby, M. foliolosa Benth., M. heringeri Barneby, M. melanocarpa Benth., M. neptuniodes Harms, M. setosa Benth. var. urbica Barneby, M. setosissima Taub., M. somnians Humb. \& Bonpl. var. viscida (Willd.) Barneby, M. splendida Barneby, M. ulei Taub. var. ulei], 2 (M. pycnocoma Benth. and $M$. cisparanensis Barneby) are tetraploid and in 3 species intraspecific variability for chromosome number (diploid and tetraploid accessions) was verified: $M$. pigra L. var. dehiscens (Barneby) D.Glazier \& Mackinder, M. setosa Benth. var. paludosa (Benth.) Barneby, and M. somnians Humb. \& Bonpl. ex Willd., all with $2 n=26$ and $2 n=52$ chromosomes.

Of the 19 species (24 accessions) studied from section Mimosa, 11 are diploid [M. affinis B.L.Rob., M. albida Humb. \& Bonpl. ex Willd. var. albida, M. debilis Humb. \& Bonpl. ex Willd., M. debilis var. debilis, M. debilis var. vestita (Benth.) Barneby, M. hypoglauca Mart. var. hypoglauca, M. papposa Benth. var. papposa, M. polycarpa Kunth var. subandina Barneby, M. polydactyla Humb. \&
Bonpl. ex Willd., M. radula Benth., M. sensitvia L. var. sensitiva, M. ursina Mart., M. virgula Barneby), 7 are tetraploid (M. acutistipula Benth. var. acutistipula, M. goldmanii B.L.Rob., M. niederleinii Burk., M. nuda Benth. var. nuda, M. pudica L., M. pudica L. var. hispida Brenan, M. skinneri Benth. var. skinneri, M. velloziana Mart.) and 1 is triploid (M. sparsiformis Barneby). No ploidy level above the tetraploid was found in the material analysed here (Table 1).

\section{Discussion}

This study doubles the number of Mimosa species with chromosome number determinations, from less than $10 \%$ to more than $20 \%$ of all taxa including accessions from all five sections and covering a broad taxonomic and geographical range when compared with previous studies (Table 1), therefore representing a significant contribution to the cytotaxonomy of this important legume genus.

With ca. 145 taxa analysed so far (Table 1; Federov 1969; Alves and Custódio 1989; Seijo 1993, 1999, 2000; Seijo and Fernández 2001; IPCN 2009), ca. 78\% of the Mimosa species are diploid, ca. $17 \%$ are tetraploid, rather similar to what we have found, ca. $3 \%$ octaploid and ca. $2 \%$ other chromosome numbers. According to these data, chromosome number cannot be considered a clear cut cytotaxonomic diagnostic character in the genus, as no particular chromosome number is restricted to any of Barneby's (Barneby 1991) sections, and polyploids are present in all sections, except Mimadenia, where only one species was examined.

The number of species we analysed in each section, 1 out of 15 in Mimadenia (6\%), 41 out of 190 in Batocaulon (21\%), 18 out of 78 in Habbasia (23\%), 4 out of 26 in Calothamnos (15\%) and 19 out of 177 in Mimosa (11\%), can be considered representative for each section, except for Mimadenia and Mimosa which were undersampled. When these data are joined to information from the literature, the percentage of studied species remains the same for Mimadenia (6\%, only one species) and increases to $27 \%$ in Batocaulon, $28 \%$ in Habbasia, 23\% in Calothamnos and $20 \%$ in Mimosa. In all sections diploid species predominate, and tetraploid taxa are found in all but Mimadenia, but are apparently more frequent in sections Calothamnos and Mimosa. Octaploids have been found in sections Calothamnos and Mimosa and the only triploid (M. sparsiformis) so far reported along with a hexaploid accession of $M$. pudica is from section Mimosa.

The chromosome numbers reported here plus those of literature (Federov 1969; Alves and Custódio 1989; Seijo 1993, 1999, 2000; Seijo and Fernández 2001; IPCN 2009) lead to the conclusion that the majority of Mimosa species and taxa are diploid, but polyploidy has played a role in the 
genus evolution and speciation. Comparing our results with recent molecular phylogenetic studies (Simon 2009; Simon et al. 2009), we found that polyploid species occur in different parts across the Mimosa phylogeny. This suggests that duplication of chromosome numbers evolved several times in the genus and that polyploidy is not restricted to any particular clade within Mimosa. On the contrary, it seems that polyploids arose independently from ancestors with lower ploidy levels and are present in divergent lineages in the genus.

Regarding intraspecific variability in chromosome numbers, published data indicate variability (sometimes related to varieties) for $M$. pudica, M. balansae, M. debilis, M. somnians, M. pigra and M. setosa. These initial reports are amplified here, where more accessions were analysed for some species. For example, for M. pigra var. dehiscens, we analysed five accessions from different localities, four from Brazil (São Paulo, Mato Grosso, and Goiás at Caldas Novas and Cristalina) and one from Bolivia (Table 1). Three Brazilian accessions were tetraploid and the Bolivian plus the Cristalina accessions were diploid. No obvious relation between geographical origin and ploidy level has been found. Seijo (1999) found intraspecific variability for two Argentinian accessions of M. pigra, a diploid one of M. pigra var. dehiscens and a tetraploid one for M. pigra var. pigra. For $M$. setosa var. paludosa studied in this paper, three tetraploid accessions were found (one from São Paulo and the others from Distrito Federal) (Table 1) and one diploid was also collected in Distrito Federal. For M. somnians, the accessions collected in Mato Grosso were diploid and the one from Rio Grande do Norte was tetraploid (Table 1). Seijo $(1993,2000)$ also reported diploid and tetraploid accessions in M. somnians. Seijo (2000) reported $2 n=26$ for $M$. debilis var. vestita collected in Paraguay and $2 n=52$ for $M$. debilis var. debilis from Argentina (Seijo 1993, 1999). Finally, for M. pudica most of the authors found tetraploidy, in accordance with our results (Table 1), but there are reports of $2 n=44$ and $2 n=78$ chromosomes, besides the most common $2 n=52$ (IPCN 2009; Seijo 1999). It is notable that polyploidy and infraspecific variability in chromosome number are often associated with widespread polymorphic species or species complexes, such as $M$. setosa or the $M$. debilis/M. nuda alliance.

The extent of intraspecific variability in chromosome numbers in the genus Mimosa still remains to be determined, due to rather limited sample sizes and rather restricted collection sites. In order to resolve that, intensive collections of several populations throughout the area of distribution of the species should be made.

According to Stebbins (1971), polyploids generally have a wider geographic distribution, being very good colonisers of new habitats and maybe more aggressive than their diploid relatives. It is striking that at least seven of the known Mimosa polyploids, M. debilis, M. nuda, M. pigra, M. pudica, M. candollei, M. somnians and M. velloziana, are weedy opportunistic species that are geographically widespread across the neotropics, often locally abundant, forming dense thickets on disturbed ruderal sites. Two of these, M. pigra and M. pudica, are amongst the world's most aggressive invasive weeds and are now naturalised and forming damaging invasive thickets across extensive parts of Africa, India and South-east Asia.

Another possible relation is that of polyploidy and higher latitudes. Seijo and Fernández (2001), studying species from southern South America, suggested that higher ploidy levels were found in higher latitudes. Among the species collected in northern Argentina between latitudes $27^{\circ} \mathrm{S}$ and $29^{\circ} \mathrm{S}$, M. vellosiella Burk., M. oligophylla Micheli and M. flagelaris Benth. were diploid, M. velloziana was tetraploid, one accession of $M$. balansae Micheli was diploid and the other tetraploid. Between $32^{\circ} \mathrm{S}$ and $35^{\circ} \mathrm{S}$, of the three species studied, M. sprengelli DC. and $M$. ramulosa Benth. were octaploid and $M$. adpressa Hooker \& Arnott was tetraploid. M. rocae Lorentz \& Niederlein collected at $38.5^{\circ} \mathrm{S}$ was also octaploid. Examining Table 1, it can be seen that of the four species collected in Rio Grande do Sul, southern Brazil (ca. $29^{\circ} \mathrm{S}$ ), three, M. incana, M. niederleinii and M. scabrella, are tetraploid and $M$. sparsiformis is triploid. The tetraploid M. biuncifera was collected in Arizona, U.S.A. at $31^{\circ} \mathrm{N}$. A few polyploid species have also been found at lower latitudes (Table 1), including M. cisparanensis, M. velloziana, M. pycnocoma, M. pudica and tetraploid accessions of M. pigra.

All these data suggest a possible relation among geography, species distribution, polyploidy and invasiveness in Mimosa, however, many more chromosome counts of a wider range of species and accessions, from other localities and especially higher latitudes, are needed before firm conclusions about the relationships can be drawn.

Acknowledgments We thank CNPq (Conselho Nacional de Desenvolvimento Científico e Tecnológico, Brazil) for a $\mathrm{PhD}$ grant to the first author and financial support and the Millenium Seed Bank of the Royal Botanic Gardens, Kew, Euan James, Sergio de Faria, Joan Sutherland, Tiina Sarkinen, Carolyn Proença, Rosaura Grether, Sara Camargo-Ricalde, John Wood, Chris Fagg and Luciano de Queiroz for provision of samples and assistance in the field.

\section{References}

Alves MAO, Custódio AVC (1989) Citogenética de leguminosas coletadas no estado do Ceará. Rev Bras Genet 12:81-92

Barneby RC (1991) Sensitivae censitae. A description of the genus Mimosa Linnaeus (Mimosaceae) in the New World. Mem N Y Bot Gard 65:1-835 
Bessega C, Hopp HE, Fortunato RH (2008) Toward a phylogeny of Mimosa (Leguminosae: Mimosoidae): a preliminary analysis of southern south American species based on chloroplast DNA sequence. Ann Missouri Bot Gard 95:567-579

Camargo-Ricalde SL, Grether R, Martínez-Bernal A, García-García V, Barrios del Rosal S (2001) Especies útiles del género Mimosa (Fabaceae-Mimosoideae) en México. Bol Soc Botan Mex 68:33-44

Caramori PH, Androcioli Filho A, Leal AC (1996) Coffee shade with Mimosa scabrella Benth. for frost protection in southern Brazil. Agrof Syst 33:205-214

Dhillion SS, Camargo-Ricalde SL (2005) The cultural and ecological roles of mimosa species in the Tehuacan-Cuicatlan Valley, Mexico. Econ Bot 59:390-394

Du Puy DJ, Labat J-N, Rabevohitra R, Villiers J-F, Bosser J, Moat J (2002) The Leguminosae of Madagascar. Royal Botanic Gardens, Kew

Federov A (1969) Chromosome numbers of flowering plants. Academy of Sciences of the USSR, Leningrad

Goldblatt P (1981) Cytology and the phylogeny of the Leguminosae. In: Polhill RM, Raven PM (eds) Advances in legume systematics, vol I. Royal Botanic Gardens, Kew, pp 263-427

Lewis G, Schrire B, Mackinder B, Lock M (2005) Legumes of the world. Royal Botanic Gardens, Kew

Lorenzi H (2000a) Árvores brasileiras: manual de identificação e cultivo de plantas arbóreas nativas do Brasil. Instituto Plantarum, Nova Odessa

Lorenzi H (2000b) Plantas daninhas do Brasil. Instituto Plantarum, Nova Odessa

Lowe S, Browne M, Boudjelas S, De Poorter M (2000) 100 of the world's worst invasive alien species: a selection from the global invasive species database. The World Conservation Union (IUCN), Auckland
Missouri Botanical Garden (2009) IPCN (index to plant chromosome numbers). http://mobot.mobot.org/W3T/Search/ipen.html. Acessed December 2009

Polhill RM, Raven PH, Stirton CH (1981) Evolution and systematics of the Leguminosae. In: Polhill RM, Raven PH (eds) Advances in legume systematics, vol 1. Royal Botanic Gardens, Kew, pp 1-26

Rivera-Arce E, Chávez-Soto MA, Herrera-Arellano A, Arzate S, Agüero J, Feria-Romero IA, Cruz-Guzmán A, Lozoya X (2007) Therapeutic effectiveness of a Mimosa tenuiflora cortex extract in venous leg ulceration treatment. J Etnopharmac 109:523-528

Seijo G (1993) Números cromosómicos en especies Argentinas del género Mimosa (Leguminosae). Bol Soc Argent Bot 29:219-223

Seijo G (1999) Chromosome studies in Argentinean species of Mimosa. Cytologia 64:241-246

Seijo G (2000) Números cromosómicos de especies de Mimosa (Leguminosae) de Paraguay. Bonplandia 10:163-167

Seijo G, Fernández A (2001) Chromosome numbers of some southernmost species of Mimosa L. (Leguminosae). Cytologia 66:19-23

Simon MF (2009) Systematics and evolution of Mimosa L. (Leguminosae) and the assembly of a Neotropical plant diversity hotspot. PhD Thesis, Oxford University, London

Simon MF, Proença C (2000) Phytogeographic patterns of Mimosa (Mimosoidae, Leguminosae) in the Cerrado biome of Brazil: an indicator genus of high-altitude centers of endemism? Biol Conserv 96:279-296

Simon MF, Grether R, Queiroz LP, Skema C, Pennington RT, Hughes CE (2009) Recent assembly of the Cerrado, a neotropical plant diversity hotspot, by insitu evolution of adaptations to fire. Proc Nat Acad Sci USA 106:20359-20364

Stebbins GL (1971) Chromosomal evolution in higher plants. Addison-Wesley, Reading 